Meta

Journal des traducteurs

Translators' Journal

\title{
Les horizons de la terminotique
}

\section{Daniel Gouadec}

Volume 32, numéro 2, juin 1987

Vers l'an 2000. La terminotique, bilan et prospectives

Objectives: Year 2000 Terminotics. State of the Art, Prospects for the Future

URI : https://id.erudit.org/iderudit/002944ar

DOI : https://doi.org/10.7202/002944ar

Aller au sommaire du numéro

Éditeur(s)

Les Presses de l'Université de Montréal

ISSN

0026-0452 (imprimé)

1492-1421 (numérique)

Découvrir la revue

Citer cet article

Gouadec, D. (1987). Les horizons de la terminotique. Meta, 32(2), 130-138.

https://doi.org/10.7202/002944ar

Ce document est protégé par la loi sur le droit d'auteur. L'utilisation des services d'Érudit (y compris la reproduction) est assujettie à sa politique d'utilisation que vous pouvez consulter en ligne.

https://apropos.erudit.org/fr/usagers/politique-dutilisation/ 


\title{
LES HORIZONS DE LA TERMINOTIQUE
}

\author{
DANIEL GOUADEC \\ Université de Rennes 2, Rennes, France
}

Le rêve électronique de tout responsable de service de traduction n'est sans aucun doute guère éloigné du système expert ou système intelligent susceptible de gérer la totalité des processus conduisant à la traduction dite "de qualité ». Dans cette perspective, la machine assurerait la totalité des fonctions de documentation, analyse, transfert, et rédaction selon les directives données, en chaque " point de décision ", par l'homme qui, simultanément, serait appelé à effectuer les choix ultimes. L'homme ne serait alors qu'un aiguilleur et contrôleur précisant les critères de recherche et transformationtraitement des données, prenant les décisions " éclairées " par la machine, et nourrissant cette machine. Car, en dernière analyse, l'ordinateur ne peut donner que ce que l'imagination et le travail de l'homme le rendent capable de donner et, si le rêve électronique doit devenir réalité (morose ?...), il reste aux terminoticiens (sic) un long chemin à parcourir.

Indiscutablement, la terminotique ou terminologie informatisée (à gestion automatique) ne peut qu'occuper une place de choix dans tout système d'aide à la traduction/documentation/rédaction destiné à faciliter (et, peut-être, enrichir) les tâches du traducteur ou rédacteur ou documentaliste. La terminotique générera des outils de plus en plus performants à condition que l'on accepte, d'une part, de remettre en cause certaines pratiques professionnelles et, plus encore, les fonctions actuelles de la terminologie. La remise en cause semble inévitable de par la nature même de l'outil informatique et il n'est guère certain que les praticiens aient à s'inquiéter de perspectives qui verraient se substituer à eux la machine "intelligente».

\section{BANQUES ET BASES DE DONNÉES TERMINOLOGIQUES}

Nous connaissons de la terminotique flambant neuve - à l'échelle historique les banques de données terminologiques et, de plus en plus souvent, les logiciels d'aides à la traduction dont nous connaissons aussi, hélas, les coûts et les limites.

L'apport fondamental des banques ou bases de terminologie réside essentiellement dans les possibilités de mise à jour rapide, de multiplication des indexations, et de " rentabilisation " des consultations. Mais peut-être a-t-on, par nécessité immédiate et manque de moyens, asservi quasi-totalement la réalisation des banques et bases de terminologie aux préoccupations des traducteurs. Entendons par là que les contenus des banques et bases de terminologie sont autant de réponses directes aux problèmes non moins directs d'appariements (l'utilisation ou interrogation présupposant la connaissance de l'objet d'interrogation réduit à l'unité terminologique). Bien entendu, pareille affirmation demeure, pour une part, caricaturale. Tout concepteur ou exploitant de banque ou base de données terminologiques saura faire valoir que les systèmes informatiques utilisés permettent, selon les cas, la constitution de sous-dictionnaires ou la recherche d'informations liées, dans le champ des fiches, à l'objet d'interrogation ou encore, quoique moins fréquemment, les interrogations "pointues" à partir d'indices croisés permettant de prémobiliser l'ensemble des termes et concepts POTENTIELLEMENT nécessaires au traducteur ou rédacteur compte tenu de l'objet de son texte. 
Un double dérapage vers le gigantisme a longtemps caractérisé la réalisation de banques et bases de données terminologiques. Le gigantisme fut d'abord le syndrome d'une lutte d'influence ou d'une course à la plus grande banque de données terminologiques. Reconnaissons qu'il fut aussi - à ce niveau - l'expression du souci légitime de rentabiliser des systèmes informatiques relativement complexes et, donc, coûteux. Et, puis le micro-ordinateur n'était pas né... Le gigantisme fut ensuite le résultat d'une préoccupation légitime de renforcement du rendement des banques et bases de données terminologiques. Il n'est plus, ici, question du nombre des enregistrements ou de la quantité d'information contenue dans chaque enregistrement, mais du nombre des constituants de l'index (descripteurs ou clés) remplissant les fonctions de tri. La terminologie, en devenant automatique et, partant, terminotique, devenait thématique par le biais des recoupements de domaines, sous-domaines et, plus encore, de concepts.

$\grave{A}$ ce niveau, la terminotique permettait de regrouper des termes/concepts ayant entre eux des relations de champ terminologique et, plus tard, des relations de champ fonctionnel. Ainsi, telle forme d'indexation autorisait la mobilisation conjointe, autour d'un terme-vedette, de tous les termes appartenant au même domaine ou sous-domaine ET de tous les termes entrant dans des combinaisons particulières avec le terme-vedette. Ces combinaisons particulières ont d'abord été les combinaisons collocatives (expressions ayant pour " noyau " le terme sur lequel porte l'interrogation) puis, rapidement, les combinaisons que l'on peut dire "fonctionnelles" ou combinaisons générées par la grammaire "culturelle" ou ensemble de principes d'interprétation d'un "univers". Pour éclairer les débats, nous dirions que l'association de type collocatif sur un terme tel que ORDINATEUR s'apparente au schéma de ORDINATEUR DÉDIÉ AU TRAITEMENT DE TEXTE (qui devient unité terminologique) alors que l'association de type fonctionnel s'apparente au schéma ORDINATEUR // TERMINOLOGIE (pour quiconque utilise l'ordinateur comme moyen de gestion de la terminologie). Les deux types d'associations se rejoignent dans l'absolu mais divergent considérablement lorsque l'on balaie le champ fonctionnel d'un objet ou concept.

Le seul problème réel posé à ce stade est celui de la conception et de la gestion des indexations de fiches permettant d'assurer les recoupements de manière infaillible. Mais ce problème n'est pas mince. Il ne peut d'ailleurs, à notre sens, être résolu que par délimitation rigoureuse de champs fonctionnels et expérimentation systématique. Il ne peut, pour tout dire, être résolu que pour des " domaines " tenant dans les limites d'une disquette sur micro-ordinateur et, par conséquent, gérés sur un petit système. Sinon, les intersections de descripteurs ou clés deviennent tellement nombreuses que les " bruits " (informations parasites) à l'interrogation interdisent toute utilisation réelle.

Faut-il voir dans les micro-bases de données terminologiques une réponse idéale aux problèmes de terminologie? Certes pas, lorsque la base doit servir des utilisateurs dont les préoccupations et champs de référence sont diversifiés. Cependant, les microbases de terminologie offrent indiscutablement des avantages dans des situations spécifiques dont nous pouvons tenter de définir les paramètres.

\section{EXTENSIONS DES TERMINOLOGIES : CHAMPS ET FONCTIONS}

Les micro-banques ou bases de terminologie apportent un moyen idéal de recherche et d'approfondissement de la réflexion sur la thématisation des terminologies et sur les principes et méthodes de l'indexation des fiches visant à permettre une "recherche terminologique absolue» sur tel ou tel concept ou terme.

Nous avons précédemment évoqué l'hypothèse d'indexations affinées rejoignant et dépassant le système des thésaurus grâce auxquels les notions obéissent à des classements rigoureux. Ces indexations affinées ne sont possibles que pour des champs clos, mais les juxtapositions ou enchâssements de champs clos finissent par aboutir à des ex- 
plorations de sous-domaines puis de domaines complexes ou étendus. Elles permettent surtout les synthèses par tris fondés sur un critère d'intersection donné entre termes/ concepts : identité, altérité, identités partielles, relations de terme générique à terme spécifique - et inversement -, relations de partie à tout, relations de cause/présupposé/ antériorité à conséquence/implication/postériorité, relations de juxtaposition ou subordination, relations de processus à appareil ou matériel, relations de processus à initiateur de processus, relations de processus à résultat de processus, etc. Les relations doivent, pour un corpus donné, être exhaustives (ou épuisées). Il sera ainsi possible, par la gestion informatique des données terminologiques, de dresser un état des algorithmes de la grammaire culturelle présidant à la mise en place de schémas de traitement ou interprétation d'un champ de l'activité humaine (à condition, comme nous l'avons dit, que le champ soit strictement délimité). Le jeu en vaut sans doute la chandelle, même si l'on dépasse alors, du moins en apparence, les préoccupations immédiates de tout traducteur par exemple. Encore faudrait-il s'interroger sur ce que sont, ou devraient être, ces préoccupations immédiates du traducteur, car chacun sait qu'il ne suffit hélas pas de connaître les " équivalents " pour traduire : il faut aussi savoir comment "fonctionne " le terme dans la langue et comment "fonctionne " le concept ou l'objet désigné dans la pratique et dans l'expérience.

S'il n'est de terminologie véritablement utile (au sens où elle simplifie les tâches du praticien) que si elle est thématique, l'informatique offre de vastes possibilités - que ce soit sur micro-ordinateur ou sur gros système. La thématisation "fonctionnelle » des terminologies peut dépasser le premier stade des indexations cumulatives évoqué plus haut. Nous envisageons, pour notre part, plusieurs orientations possibles.

\section{Extensions linguistiques textuelles}

Si la thématisation des termes et concepts paraît nécessaire, elle doit s'orienter dans deux directions : la thématisation "linguistique " et la thématisation "fonctionnelle".

La thématisation linguistique recouvre l'ensemble des procédures par lesquelles chaque unité terminologique se trouve accompagnée de ses blocs phraséologiques (stéréotypies, collocations, combinaisons linguistiques standards ou non standards dont le terme-vedette forme le noyau). La première forme de thématisation est une thématisation a posteriori permise par la gestion informatique. L'outil est le système permettant la recherche de la vedette (et de son environnement linguistique) dans le champ de toute fiche. En d'autres termes, cette recherche est celle par laquelle la " machine " extrait de l'ensemble des données entrées la totalité des combinaisons linguistiques dans lesquelle figure le terme-vedette ou objet de l'interrogation.

Mais cette possibilité offerte par la machine doit aussi conduire à définir, systématiquement, pour tout travail terminologique, un objectif d'expansion progressive des unités traitées. Ainsi, la terminologie peut " grandir " pour devenir une phraséologie ou recueil de toutes expressions dans lesquelles figurent des unités terminologiques. Ces unités phraséologiques seraient traitées comme le sont, actuellement, les unités terminologiques. Peut-être verrions-nous émerger, si cette proposition se confirmait, une phraséotique venant compléter la terminotique...

Former et traiter des unités phraséologiques dont l'exploitation serait d'une facilité déconcertante - puisque la gestion par informatique permettrait une interrogation à partir de n'importe quel élément ou mot figurant dans les unités phraséologiques peut contribuer à faciliter la tâche du traducteur comme du rédacteur. Et, si pareille hypothèse semble séduisante, il faut considérer que les limites de l'unité phraséologique ne sont pas absolues. 
De la phraséologie définie comme traitement de " locutions " ou expressions intégrant des unités terminologiques, on passe sans peine à des unités de très large portée qui ne vont pas sans poser des problèmes de traduction. Nous pensons ici à des stéréotypes en une ou plusieurs phrases, voire en un ou plusieurs paragraphes dont les récurrences, dans un même texte ou dans des textes différents correspondent à autant de modalités d'organisation ou structuration du texte. À titre d'exemple, considérons un texte portant sur un logiciel informatique et dans lequel se répète le bloc «ENTER $C / R T O$ SUBSTITUTE DEFAULT VALUE TO EXISTING CODES ». L'exemple peut sembler hautement spécifique mais le libellé (ou un libellé approchant) est fréquent dans certains types de textes. Ne pourrait-on alors concevoir que des "formulations " de ce type, atteignant parfois une longueur considérable soient consignées dans des dictionnaires automatiques... comme elles le sont dans le glossaire du système de traitement de texte qu'utilise le traducteur " traitant " précisément ce type de récurrences.

Ne pourrait-on pas, simplement, pour commencer par le commencement, imaginer un système reposant sur ce principe et portant sur le domaine des articulations générales d'un texte ou de textes (en reprenant l'opposition entre le dictionnaire général et les dictionnaires spécialisés) ? On obtiendrait ainsi un "dictionnaire automatique " ou une base de données d'aides à la traduction et à la rédaction, grâce à laquelle, par exemple, le traducteur ou rédacteur disposerait, pour chaque type de relation intratextuelle, d'un vaste éventail de schémas d'expression. Ainsi peut-on mettre à la disposition des praticiens (débutants) des schémas linguistiques et rhétoriques d'expression de restrictions, ou d'exemples, ou de transitions, etc. et, sans aucun doute, aller jusqu'à "mettre en machine" les stéréotypes de l'organisation du discours scientifique ou du discours technique (avec spécialisations progressives). À titre d'exemple, rien n'interdit d'imaginer que le traducteur ou rédacteur dispose, en tapant EXEMPLE et PHYSIQUE et SPÉCIALISÉ, d'une batterie de formulations utilisées dans telle langue (et culture) pour donner un exemple dans un texte spécialisé de physique. Il reste simplement à construire les banques de données qui ne seraient guère éloignées, dans le principe, des banques de données terminologiques. La thématisation linguistique des unités terminologiques atteindrait alors son degré maximal.

Extensions fonctionnelles

Reste la thématisation "fonctionnelle ". Ici encore, l'outil informatique s'avère précieux dans la mesure où il ouvre des perspectives. La thématisation fonctionnelle est possible selon plusieurs voies.

En premier lieu, rappelons que les interrogations automatiques fournissent, lorsque l'indexation des fiches est adéquate, les cooccurrents fonctionnels de tel terme : termes/concepts liés, dans l'expérience ou l'activité considérée, au terme/concept sur lequel porte l'interrogation.

En second lieu, l'outil informatique ne se limite pas à la base de données multicritères qui constitue cependant, actuellement, le modèle le plus répandu (si l'on excepte le fichier automatique pour lequel la recherche n'est guère possible qu'à partir d'une clé unique constituée par le terme lui-même ou par l'un des termes appariés ou par l'un des champs de la fiche). La recherche multicritères dans une base du même nom est une recherche à partir de clés combinées (et toujours multiples). Son rendement est, par définition, plus important que celui de la recherche sur terme ou champ unique. Mais la thématisation vraie intervient lorsque les termes sont «traités 》 selon des schémas de bases arborescentes que l'on peut parcourir non plus pour connaître des appariements, définitions, ou données "figées" dans un champ de fiche terminologique, mais pour savoir quelle place, fonctions, et modalités de traitement sont celles du concept dans une expérience ou activité donnée. La différence entre la base de données standard et la base ar- 
borescente est parallèle à la différence entre, disons, le dictionnaire du traitement de texte et le cours portant sur ce même traitement de texte. L'avantage du traitement automatique des données réside dans le fait que l'indexation des fiches de l'arborescence contribue à simplifier les accès à l'information voulue. On peut ainsi obtenir directement les informations sur un point donné pour ensuite remonter ou redescendre les ramifications de l'arborescence jusqu'à ce que l'on ait obtenu le type et la quantité d'informations nécessaires.

Bien entendu, le mieux n'étant pas - en la circonstance - l'ennemi du bien, rien n'interdit de fusionner les deux types de bases de données pour combiner - par transposition - dictionnaire et encyclopédie et obtenir un parcours optimal dans une base de savoirs qui ne sont plus strictement terminologiques... ou qui commencent à le devenir si l'on accepte de définir la terminologie comme un point de rencontre entre des savoirs linguistiques et des savoirs "culturels-fonctionnels».

Pareil outil permettrait à tout utilisateur de se documenter pleinement sur un SUJET et non plus sur une succession d'OBJETS. La documentation serait accélérée puisque le parcours documentaire serait assuré par la "machine». Il reste simplement à construire l'outil terminologique puisque l'outil informatique existe depuis longtemps. Mais ce ne sera pas une mince affaire que de constituer la donnée terminologiqueencyclopédique qui, par parenthèse, devra nécessairement inclure les données phraséologiques et les données d'expression précédemment évoquées au titre de la thématisation linguistique. La question posée est simple : est-il besoin d'un outil informatique pour gérer ce qui, en apparence, n'est rien d'autre qu'un manuel accompagné de son glossaire ? Quiconque connait les possibilités de la gestion informatique sera sans doute convaincu que oui. Quiconque craint les incidences de l'informatisation sur la fonction de rédaction et/ou traduction penchera - légitimement - vers le support papier ou, sans doute, vers une position sereine s'appuyant sur le fait que le traducteur "se débrouille assez bien comme cela " et que, de toute façon, il n'appartient pas au terminologue de se préoccuper de ce genre de choses. Mais la question des limites des fonctions ou attributions du terminologue n'en demeurera pas moins ainsi posée par les perspectives ouvertes par ce type d'outil en matière de documentation et d'autoformation : si le terminologue n'a pas à se préoccuper de "construire des manuels" - automatiques ou non - rien n'interdit de penser qu'il se trouvera des " auteurs de manuels " devenant terminologues et exploitant l'outil informatique pour offrir à tout le monde (et surtout aux professionnels de la pratique linguistique au nombre desquels comptent les traducteurs) des "extensions» terminotiques.

\section{Extensions graphiques}

Au titre des "extensions terminotiques", on peut envisager de mettre en œuvre l'outil informatique pour réduire les degrés d'ambiguïté linguistique. Si l'on accepte de considérer que les unités terminologiques et phraséologiques sont la représentation linguistique d'un " morceau d'univers ou expérience ", ces unités sont corrélées à l'ensemble des concepts et intersections entre concepts constituant le fragment d'univers ou expérience. Or, chacun sait que les représentations linguistiques ne sont guère exemptes d'ambiguités. Pourquoi, dans ce cas, ne pas concevoir des banques ou bases de données terminologiques-encyclopédiques illustrées selon des systèmes de pilotage automatique ? Dans cette perspective, chaque fiche serait accompagnée d'une ou plusieurs représentations graphiques ou vidéographiques illustrant ou représentant directement l'objet documentaire. L'explication ou interprétation deviendrait alors absolue.

L'hypothèse la plus basse d'extension graphique concerne le pilotage, par l'ordinateur, d'une base de données sur diapositives comportant schémas, photographies, et enchaînements décrivant les processus. L'hypothèse moyenne concerne la gestion gra- 
phique par programme informatique (schémas et animations réalisés en logiciel et s'affichant sur l'écran du terminal). L'hypothèse haute concerne la base de données vidéographiques sur vidéodisque. Dans ce dernier cas, les possibilités d'illustration deviennent considérables dans la mesure où les vidéodisques standards permettent l'enregistrement de plus de 100000 images et, naturellement, la création, à partir de ces images, de combinaisons quasi infinies de scénarios illustrant les processus. Les temps de recherche sur vidéodisque étant comparables aux temps de recherche des données textuelles (fiches), les affichages seraient simultanés (et l'on surmonterait ainsi directement les problèmes que pose la gestion d'une base de données de diapositives pour laquelle les temps d'affichage tendent à induire des décalages entre l'affichage de la donnée textuelle et l'affichage de la donnée graphique). L'idéal serait, ici, que le vidéodisque utilisé soit techniquement conçu de manière à permettre l'enregistrement d'images ou séquences d'images "à domicile » : vidéodisque réinscriptible permettant à l'utilisateur de constituer sa base d'images sans devoir faire appel au secteur industriel pour pressage d'un disque dont les contenus seraient figés une fois pour toutes.

\section{CONCLUSIONS ET STATUT DES SYSTÈMES TERMINOTIQUES "AUGMENTÉS "}

La question fondamentale que l'on peut poser à ce stade concerne le caractère d'" utilité " de systèmes terminotiques dont les extensions linguistiques seraient telles que ces systèmes en viennent à inclure : $a$ ) les unités phraséologiques automatiquement mobilisées lorsque l'interrogation porte sur l'un de leurs constituants, $b$ ) les unités d'organisation textuelle ou unités de structuration d'un discours spécifique puis $c$ ) inclure des représentations graphiques ou vidéographiques des concepts et intersections de concepts formant l'opérande et les algorithmes d'une grammaire culturelle ou expérientielle (algorithmes d'interprétation et communication d'une expérience humaine). La réponse ne peut guère être une réponse par oui ou par non.

En premier lieu, répondre catégoriquement non c'est réduire la terminologie à un rôle exclusif d'outil de la traduction. La terminologie est aussi objet et outil d'apprentissages de type culturel (en formation initiale comme en formation continue). La terminologie est encore la partie émergée d'ensembles cohérents, complexes (ensembles linguistiques et culturels ou expérientiels) dans lesquels elle vient se replacer dans toute situation de communication active (rédaction/traduction/expression) ou passive (compréhension ou interprétation). Il semblerait donc tout naturel de mettre à profit les possibilités de l'outil informatique pour tenter une réintégration de l'unité terminologique à l'ensemble de ses champs fonctionnels (linguistiques-phraséologiques ou culturelsexpérientiels). Tant que les réalisations envisagées ci-dessus n'existeront pas, il sera difficile de porter jugement sur leur utilité. Tant que ces mêmes réalisations ne seront pas d'usage répandu, il est difficile de donner à la terminologie (ou terminotique) sa vraie place. Nous pensons que la terminologie a été "grandie " lorsqu'elle est devenue autonome et " terminologie pour la terminologie " (ou, plus précisément : terminologie pour les traducteurs). Nous pensons aussi que le mouvement inverse qui tend à se préciser et conduira à remonter vers les intégrations encyclopédiques de la terminologie constituera l'un des apports les plus significatifs de la terminotique. La terminotique marquera, à notre sens, une étape importante dans la redéfinition de la nature des données terminologiques et de leurs fonctions. Elle marquera parallèlement une étape importante, si l'évolution se confirme, dans l'évolution des fonctions des terminographes (ou terminoticiens).

Fondamentalement, si les extensions terminotiques se développent, le terminologue/terminographe/terminoticien deviendra, à court terme, un véritable spécialiste de domaines. Ses compétences ne se limiteront plus à la collation de données et à la réalisation d'appariements : elles deviendront compétences d'indexation des données et d'or- 
ganisation ou structuration des savoirs. Le terminologue/terminographe/terminoticien se trouvera, en quelque sorte, " aspiré » vers le haut par la machine qu'il lui appartiendra de nourrir : en enrichissant les programmes, il enrichira ses tâches (mais celles-ci n'en deviendront que plus exigeantes) et, surtout, il enrichira considérablement l'outil ainsi mis à la disposition de tous les praticiens de la communication linguistique et non plus des seuls traducteurs. La machine contraint l'homme à accroître sa rigueur de travail en même temps qu'elle démultiplie les possibilités de gestion des données. Il serait dommage de n'utiliser des logiciels puissants que pour reproduire (comme le font si souvent les programmes d'enseignement assisté par ordinateur) les schémas ou modèles que l'on a appris à bien maîtriser avec du papier et un crayon. À outil nouveau devrait correspondre une "prise de pouvoir de l'imagination " (à condition que ce ne soit pas seulement celle de l'imagination technocratique dont les horizons s'arrêtent à la ligne rouge d'une gestion frileuse).

Nous sommes profondément persuadés que l'informatique peut apporter énormément de choses si elle n'est pas exploitée dans les limites strictes des contraintes qu'imposaient les supports-papier. Nous pensons surtout que l'aspiration de la terminologie "dans " l'outil informatique ne tardera guère à modifier radicalement la nature et la fonction des données terminologiques trouvant très rapidement dans la terminotique le moyen de se refondre dans des unités plus grandes : combinaisons linguistiques ou d'expression et combinaisons interconceptuelles. $\mathrm{La}$ terminotique pourrait ainsi reprendre sa place au centre d'un univers ou, plus précisément, apporter les clés ouvrant, de proche en proche, des séries de portes jusqu'à ce que tout forme un ensemble de structures cohérentes. Après tout, il ne servait pas à grand chose de se battre pour que les mots cessent d'être le début et la fin de toute activité de traduction si c'était pour se contenter de créer une situation dans laquelle les termes sont "finis ", fermés, ou refermés sur leur propre nombril.

\section{LES OUTILS DE L'UTOPIE ?}

Si l'on accepte de considérer, par remise en cause "informatique » du statut de la terminologie, que l'unité phraséologique est unité de travail et que, plus encore, l'unité de structuration d'un texte (scénario ou stéréotype de formulation de relations intratextuelles) doit figurer dans les dictionnaires au même titre que le mot ou le terme, il deviendra possible de générer des outils du rédacteur et des outils du traducteur. Peut-être aboutira-t-on, rapidement, à la situation naguère jugée optimale de rédactions parallèles de textes dans l'une et l'autre langues. Rêvons un peu...

Le traducteur s'installe devant son terminal multiposte : clavier, écran découpé en fenêtres, imprimante, écran de visualisation des données graphiques-iconographiques, système de lecture optique. Il ouvre la session de travail en lançant la lecture optique du texte (ou, dans l'immédiat, sa saisie). Il peut alors, tranquillement, prendre connaissance des nouvelles du jour ou suivre son émission favorite sur l'un des écrans. Pendant ce temps, la machine effectue un tri des unités terminologiques, des unités phraséologiques, des unités d'articulation textuelle et, peut-être même, des unités de scénario que comporte le texte. Un signal sonore indique au traducteur que les tris sont effectués et qu'il peut prendre connaissance des résultats : une quantité variable de termes fait déjà l'objet d'appariements et une quantité variable d'unités phraséologiques se trouve prémobilisée en relation avec les unités terminologiques. Peut-être même la machine a-telle déjà prévu un scénario standard en français (si la traduction s'effectue vers le français) avec, le cas échéant, des ensembles de sous-scénarios intéressant telle ou telle partie du texte. Connaissant les éléments acquis et les éléments restant à acquérir, le traducteur sollicite une recherche documentaire portant sur les termes à élucider en se connectant à une base de données complémentaires. Il voit alors s'afficher à l'écran les don- 
nées (affichage en fenêtre), accompagnées d'illustrations, animations, films, etc., apportant une documentation exhaustive. Il demande l'enregistrement, dans son propre programme de travail, des données qui lui semblent pertinentes ou potentiellement utiles. Pour ce qu'il lui manque encore, il génère une demande (par télématique) adressée à un centre de documentation spécialisée.

Le traducteur peut désormais lancer le processus de traduction. Il choisit un scénario d'organisation du texte ou d'une partie du texte : décide qu'il commencera par telle information puis continuera par telle autre, et ainsi de suite, et indique déjà la catégorie de relation entre les divers constituants ou passages : introduction + exemple + définition de tel terme + énumération des arguments + tableau de chiffres, etc. Le scénario s'inscrit, pour mémoire, dans une fenêtre (ou sur le papier par impression). L'analyseur syntaxique-terminologique-phraséologique propose des hypothèses parmi lesquelles le traducteur effectue un choix. Le logiciel de transfert effectue les transferts voulus et propose la totalité des hypothèses possibles en matière de structuration syntaxique. Si une relation interphrastique ou interparagraphique est nécessaire, le logiciel propose un choix de solutions possibles. Si une relation ou un élément n'est pas maîtrisé, le traducteur demande à reconsulter la base encyclopédique-iconographique. Le traducteur choisit alors les éléments de solution qui lui paraissent les plus satisfaisants et continue avec un bloc de travail complémentaire (phrase suivante ou paragraphe suivant). Une fois que tous les choix voulus ont été effectués, le traducteur met en route l'homogénéisateur rédactionnel qui propose des recombinaisons de l'unité textuelle transférée.

Dans le schéma envisagé ci-dessus, le traducteur continue à remplir la fonction essentielle d'aiguilleur et de juge ultime des choix. La totalité du système informatique n'a d'autre fonction que de lui apporter des hypothèses successives. Pour utopique qu'elle puisse paraître, pareille situation n'est guère différente, dans son principe de celle qu'a engendré l'invention de la calculette : la calculette effectue les opérations mais l'utilisateur décide toujours de la nature des opérandes et de la nature des opérations. Ici, le traducteur décide toujours de la nature des opérandes (sauf dans le cas d'appariements terminologiques stricts) et, plus encore, de la nature et de la séquence des opérations. La situation du traducteur trouverait d'ailleurs son pendant dans celle du rédacteur " câblé " auquel s'offriraient des scénarios de rédaction (plans) avec sous-scénarios, des ensembles de données factuelles, un vaste choix de procédés d'écriture selon le type, l'objet et le domaine du texte (et, sans doute, selon les lecteurs putatifs), un non moins vaste choix de charnières interphrastiques ou interparagraphiques et, pour en finir, un choix de "mots pour le dire ". Il resterait, lui aussi, maître à bord et assurerait le pilotage à l'instrument comme le pilote de l'avion prend des décisions en fonction des données affichées par la totalité des affichages du cockpit.

Utopie? Certes oui, mais utopie dans laquelle l'homme reste aux commandes. Coûts prohibitifs ? Peut-être ; mais ne consacre-t-on pas beaucoup d'argent à la réalisation de documents-papier dont la consultation demeure aléatoire? Chimère ? Indiscutablement ; si l'on se laisse bercer par l'illusion que la machine " pense " et si l'on substitue à l'intelligence humaine l'algorithme mécanique de l'ordinateur.

\section{CONCLUSIONS}

Nous envisageons simplement d'aller, lentement, vers l'utopie raisonnée (?! ?) en prenant appui sur ce que nous avons déjà maîtrisé (les banques et bases de données terminologiques) pour rejoindre, petit à petit, les systèmes complets d'aide à la traduction et à la rédaction. Nous envisageons ainsi de proposer une réintégration de la terminologie à sa combinatoire linguistique (phraséologique) avec recherche automatique des combinaisons. Nous envisageons aussi d'aller jusqu'au bout de ce propos en incluant dans le champ de la terminotique-phraséotique les BLOCS d'expression que constituent 
les charnières des textes et tous les stéréotypes de formulation au nombre desquels figurent les scénarios-plans de textes ou paragraphes. Nous envisageons parallèlement d'étendre le champ de la terminotique à ses relations avec l'univers interconceptuelfonctionnel-expérientiel représenté. Nous pensons que la réintégration selon cet axe peut être totale grâce aux modalités d'indexation des bases et, plus encore, grâce aux systèmes de représentation graphique ou vidéographique (en réservant une place de choix au vidéodisque qui combine les avantages de l'arrêt sur image-objet/concept et ceux de la thématisation par séquence construite). Nous pensons que, à ce stade, s'établira un pont naturel avec les systèmes d'analyse syntaxique, de transfert automatique et de génération textuelle/rédactionnelle (ces derniers restant à construire). Mais nous demeurons persuadés qu'il faudra raison garder et non pas " plaquer » sur la terminologie une gestion automatique de principe mais réfléchir d'abord aux fonctions des outils informatisés et à la nature des données. Sinon, le passage de la terminologie à la terminotique n'aura été que l'émergence d'un néologisme éphémère et l'informatique aura simplement contribué à donner des oripeaux tout neufs à une discipline trop vite rigidifiée. 